\title{
EL RELICTO GLACIAL LEISTUS (POGONOPHORUS) PUNCTICEPS FAIRMAIRE \& LABOULBÈNE, 1854 (COLEOPTERA, CARABIDAE): NUEVOS DATOS SOBRE DISTRIBUCIÓN, AUTOECOLOGÍA Y PRESENCIA EN EL MEDIO SUBTERRÁNEO SUPERFICIAL (MSS)
}

\author{
Vicente M. Ortuño ${ }^{1, *}$, Pablo Barranco ${ }^{2}$, Alberto Jiménez-Valverde ${ }^{3} \&$ Alberto Sendra ${ }^{4}$ \\ 1,3,4 Grupo de Investigación de Biología del Suelo y de los Ecosistemas Subterráneos, Departamento de Ciencias de la Vida, \\ Facultad de Ciencias, Universidad de Alcalá, 28805 Alcalá de Henares, Madrid, España. \\ VMO: vicente.ortuno@uah.es - ORCID iD: https://orcid.org/0000-0001-5734-1621 \\ AJV: alberto.jimenezv@uah.es - ORCID iD: https://orcid.org/0000-0001-9962-2106 \\ ${ }^{2}$ CECOUAL. Dpto. Biología y Geología. CITE-IIB. Universidad de Almería. Ctra. Sacramento s/n. La Cañada. 04120 Almería, \\ España. pbvega@ual.es - ORCID iD: https://orcid.org/0000-0002-9411-3991 \\ ${ }^{4}$ Colecciones Entomológicas Torres-Sala, Servei de Patrimoni Històric, Ajuntament de València, València, España. \\ alberto.sendra@uv.es - ORCID iD: https://orcid.org/0000-0002-1329-3426 \\ *Autor para correspondencia: vicente.ortuno@uah.es
}

\section{RESUMEN}

Numerosos muestreos realizados por el territorio peninsular, a lo largo de estas tres últimas décadas, han deparado el hallazgo de Leistus (Pogonophorus) puncticeps Fairmaire \& Laboulbène, 1854 en áreas geográficas en las que se desconocía su presencia hasta la fecha. Las nuevas citas amplían la distribución ibérica de la especie hacia enclaves mediterráneos meridionales, perfilando un patrón de distribución discontinuo por el tercio oriental. Al mismo tiempo, estos hallazgos revelan cierta variabilidad, muy leve, en la forma de la lámina apical del edeago. Por otro lado, se confirman los parajes quebrados y abruptos como enclaves potencialmente favorables para la supervivencia de esta especie, especialmente si disponen de laderas pedregosas. En general, las nuevas localidades en donde se ha hallado $L$. (P.) puncticeps, cuentan con precipitación escasa y torrencial, una pluviosidad media que ronda los 600-700 mm, y una notable amplitud térmica. Como novedad, y utilizando Estaciones de Muestreo Subterráneo (EMS), se ha colectado en el Medio Subterráneo Superficial (MSS). El hallazgo de esta especie en este singular medio hipogeo y en diversos enclaves ibéricos (Sierra del Moncayo, formaciones montañosas del norte de la provincia de Alicante y Sierra de María), pone de manifiesto su tendencia troglófila, y más probablemente de tipo subtroglófila. Los nuevos hallazgos, sumados a los ya conocidos, sugieren que $L$. (P.) puncticeps ha seguido la misma historia evolutiva que Leistus (Pogonophorus) montanus Stephens, 1827 y Leistus (Pogonophorus) parvicollis Chaudoir, 1869, constituyendo una triada de relictos glaciales, resultado de un proceso de especiación por vicarianza. Los cambios acaecidos en el período postglacial explicarian la distribución discontinua que muestra en la actualidad $L$. (P.) puncticeps, y su tendencia a explorar, en estos enclaves, el MSS.

Palabras clave: Leistus; Pogonophorus; Nebriini; corología; subtroglófilo; relicto glacial; Medio Subterráneo Superficial; península ibérica

\section{ABSTRACT}

The glacial relict Leistus (Pogonophorus) puncticeps Fairmaire \& Laboulbène, 1854 (Coleoptera, Carabidae): new data on distribution, autoecology, and presence in the Mesovoid Shallow Substratum (MSS)

Over the last three decades, intensive sampling of the MSS in the lberian Peninsula has revealed new records of Leistus (Pogonophorus) puncticeps Fairmaire \& Laboulbène, 1854 in previously unknown areas. These new records extend the species geographic range towards southern Mediterranean localities, showing a discontinuous distribution pattern across the eastern third of the Iberian Peninsula. Also, some slight variability in the shape 
of the apical lamina of the edeago is revealed. Abrupt landscape, especially in stony slopes, arises as a favorable habitat for the species. In general, the new localities for $L$. (P.) puncticeps have low and torrential precipitation, an average rainfall of around $600-700 \mathrm{~mm}$, and remarkable thermal amplitude. The species is collected for the first time in the Mesovoid Sallow Substratum (MSS) using subterranean sampling devices (SSD). The presence of this species in this hypogean habitat across several iberian areas (Sierra del Moncayo, mountains of the north of Alicante province and Sierra de Maria) indicates its (sub)troglophile condition. The new records, in addition to previously available data, suggest the same evolutionary history for $L$. (P.) puncticeps as for Leistus (Pogonophorus) montanus Stephens, 1827 and Leistus (Pogonophorus) parvicollis Chaudoir, 1869, being the three species glacial relicts as a result of vicariant speciation. The environmental changes that occurred during the postglacial time period could explain the discontinuous distribution that $L$. (P.) puncticeps shows at present and its tendency to occupy the MSS.

Key words: Leistus; Pogonophorus; Nebriini; chorology; subtroglophilous; glacial relict; Mesovoid Shallow Substratum; Iberian Peninsula

Recibido/Received: 26/10/2019; Aceptado/Accepted: 30/03/2020; Publicado en línea/Published online: 19/06/2020

Cómo citar este artículo/Citation: Ortuño, V. M., Barranco, P., Jiménez-Valverde, A. \& Sendra, A. 2020. El relicto glacial Leistus (Pogonophorus) puncticeps Fairmaire \& Laboulbène, 1854 (Coleoptera, Carabidae): nuevos datos sobre distribución, autoecología y presencia en el Medio Subterráneo Superficial (MSS). Graellsia, 76(1): e107. https://doi.org/10.3989/graellsia.2020.v76.255

Copyright: (C) 2020 SAM \& CSIC. This is an open-access article distributed under the terms of the Creative Commons Attribution 4.0 International (CC BY 4.0) License.

\section{Introducción}

El género Leistus Frölich, 1799 cuenta con 15 especies en la península ibérica (Serrano, 2013), de las que 12 pertenecen al subgénero nominal y 3 al subgénero Pogonophorus Latreille, 1802. Diversas especies de este género han sido colectadas de forma recurrente, y numerosa, en el Medio Subterráneo Superficial (MSS) de enclaves montañosos ibéricos (Ortuño et al., 2019; Ortuño obser. pers.). El MSS fue descubierto hace décadas por Juberthie et al. $(1980,1981)$ y, al mismo tiempo, también por Uéno (1980, 1981), si bien el nombre de "milieu souterrain superficiel", y su descripción detallada, se debe al equipo franco-rumano (Juberthie et al., 1980, 1981). Estos hábitats están formados por las fisuras e intersticios del subsuelo, constituyendo una compleja red de espacios subterráneos como resultado de la alteración físico-química de la roca madre. Se hallan cerca de la superficie, con frecuencia en contacto con los horizontes edáficos, lo que les confiere la característica de ecotono entre el medio epigeo y el medio hipogeo profundo (Moseley, 2010). La condición "superficial" de estos hábitats, propicia que sean ricos en materia orgánica y energía, provenientes de los horizontes edáficos (Gers, 1998), bien por efecto de la gravedad o por el desplazamiento vertical de fauna edáfica y endógea hacia espacios más profundos (Giachino \& Vailati, 2017). También se caracterizan por la ausencia de luz, por contener atmósferas ricas en vapor de agua y temperaturas cuyas variaciones en los ciclos día/noche, y estacionales, se ven ligeramente amortiguadas con respecto a las que experimenta el medio epigeo (Pipan et al., 2011). El MSS puede estar presente en diferentes tipos de litologías y su formación responde a distintos procesos, lo que conduce a clasificar estos hábitats en cuatro categorías principales (Juberthie et al., 1980, 1981; Oromí et al., 1986; Juberthie, 2000; Ortuño et al., 2013): MSS coluvial, MSS aluvial, MSS rocoso y MSS volcánico. Aunque el MSS está ampliamente extendido por gran parte de la corteza continental, a día de hoy, sigue siendo un hábitat muy poco explorado. Una revisión detallada sobre el MSS puede encontrarse en Mammola et al. (2016).

El objetivo de este trabajo es dar a conocer nuevos registros de Leistus (Pogonophorus) puncticeps Fairmaire \& Laboulbène, 1854 (Fig. 1), ampliando el área de distribución de esta especie $\mathrm{y}$, a partir de las observaciones realizadas sobre el hábitat que ocupa, deducir algunos de sus requerimientos ecológicos. También, se subraya el importante papel que juega el MSS en la supervivencia de esta especie, especialmente en las áreas montañosas más meridionales.

\section{Material y métodos}

Los muestreos se han realizado por todo el ámbito ibérico a lo largo de las tres últimas décadas. Se han utilizado diferentes técnicas para la colecta de entomofauna epiedáfica, edáfica e hipogea, destacando la búsqueda por rastreo, cribado de hojarasca, o la utilización de trampas pitfall, aparatos de Berlese y Estaciones de Muestreo Subterráneo (EMS). Este último procedimiento para muestrear el MSS, es la técnica de colecta que más novedades ha aportado sobre la distribución y biología de $L$. (P.) puncticeps (Fig. 1). Las EMS (Fig. 2) consisten en un cilindro de PVC ( $1 \mathrm{~m}$ de longitud y $11 \mathrm{~cm}$ de diámetro), con numerosas perforaciones de $8 \mathrm{~mm}$ de diámetro en su 


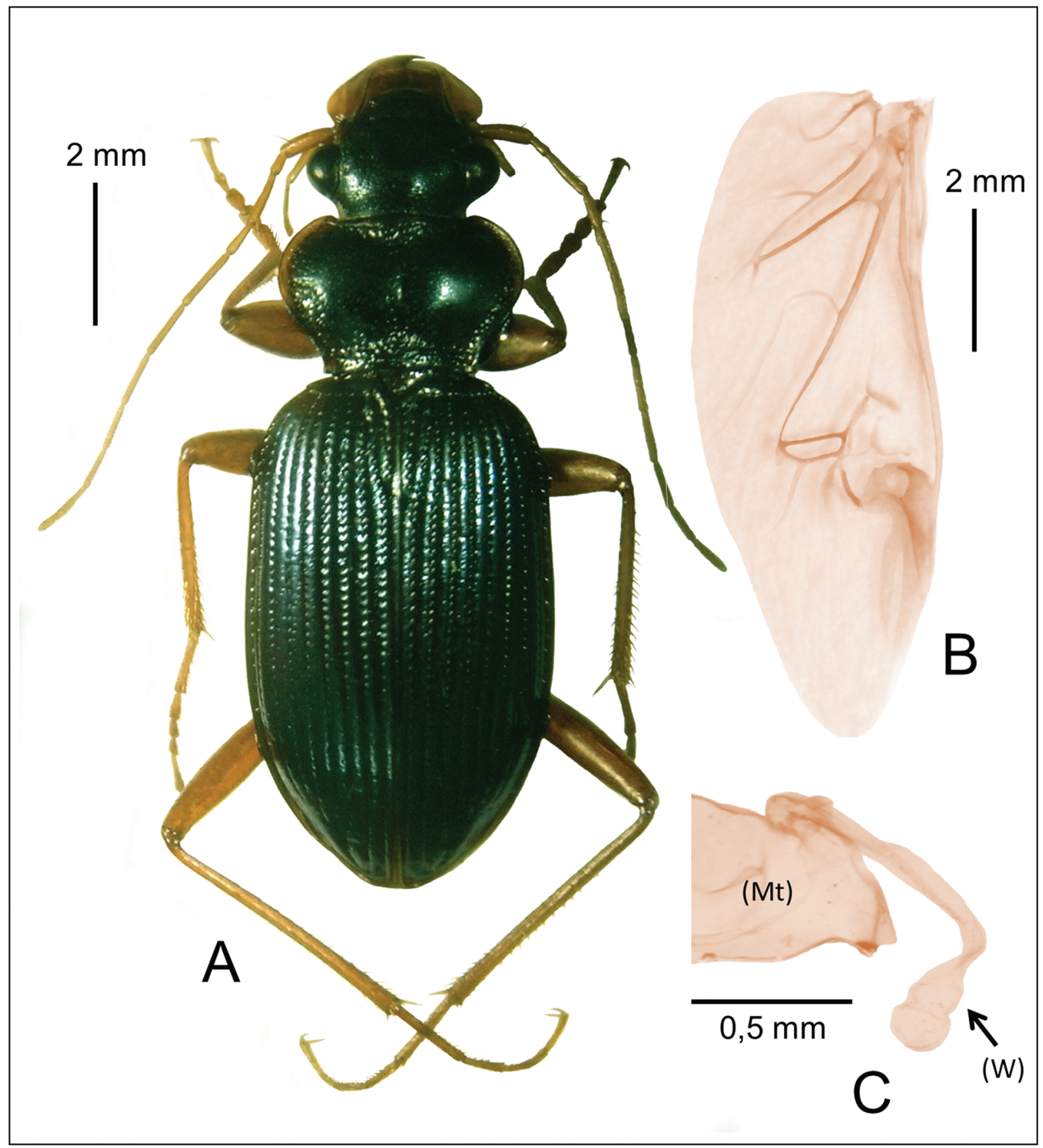

Fig. 1.- A) Imago (macho) de Leistus (Pogonophorus) puncticeps de la Sierra de María (Almería); B) Ala derecha de L. (P.) puncticeps de la Sierra del Moncayo (Zaragoza); C) Metatórax (Mt), lado derecho, y el ala (W) reducida de Leistus (Leistus) barnevillei Chaudoir, 1867 del Pico de la Devesa (León).

Fig. 1.-A) Imago (male) of Leistus (Pogonophorus) puncticeps from Sierra de María (Almería); B) Right wing of L. (P.) puncticeps from Sierra del Moncayo (Zaragoza); C) Metathorax (Mt), right side, and the reduced wing (W) of Leistus (Leistus) barnevillei Chaudoir, 1867 from Pico de la Devesa (León).

tramo inferior que, tras ser instalado verticalmente en el subsuelo, posibilitan el acceso de la fauna en una franja de profundidad de $-0,5 \mathrm{~m}$ a $-0,9 \mathrm{~m}$; por el interior del tubo se desliza, con ayuda de un hilo de nylon, una trampa pitfall que se ajusta perfectamente a su diámetro; contiene 1,2-propanodiol como líquido conservante, y queso oloroso como cebo. Los muestreos subterráneos con EMS se iniciaron en 2011 en las sierras septentrionales de Alicante, y se extendieron a otros enclaves montañosos (MSS coluvial) de 


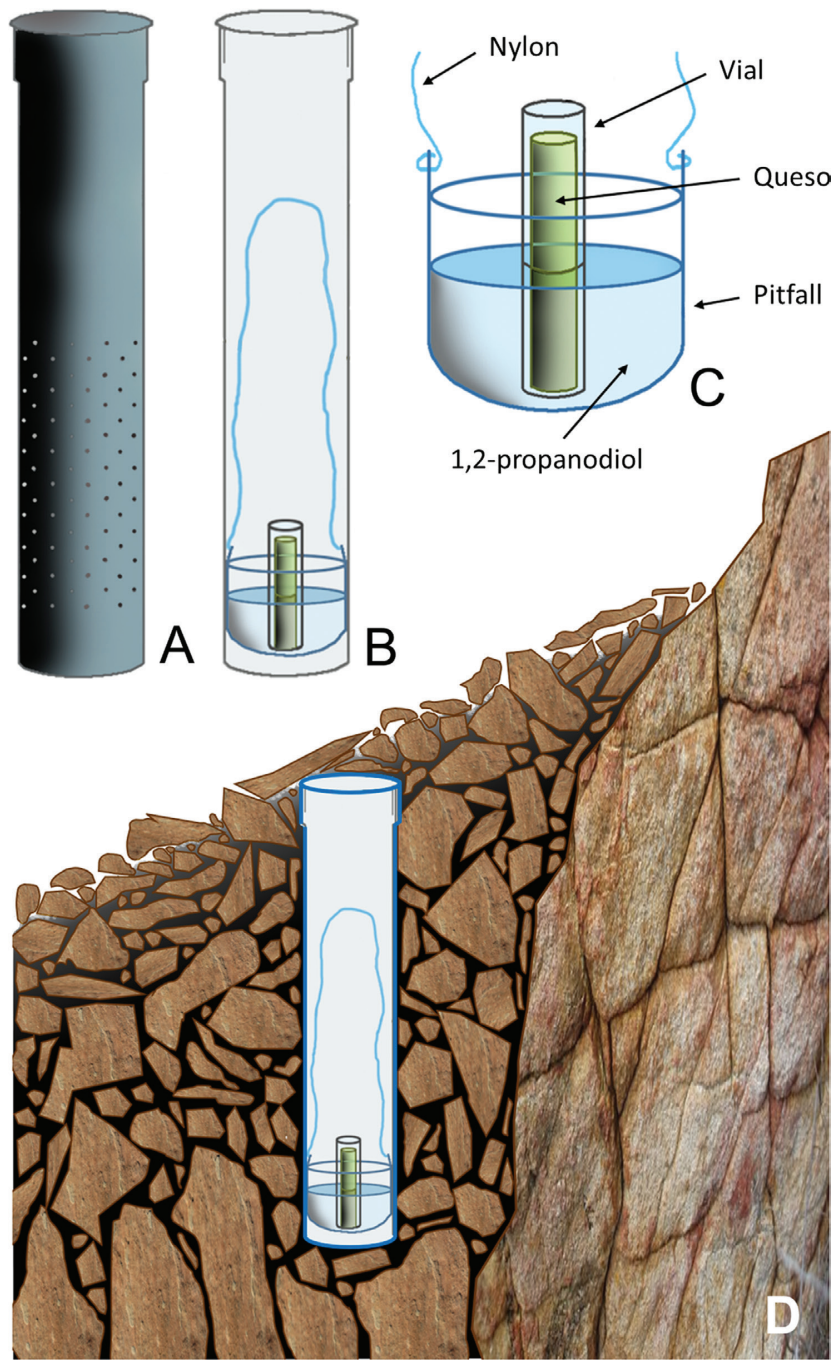

Fig. 2.- Estaciones de Muestreo Subterráneo (EMS). A) Aspecto externo; B) sección longitudinal del tubo mostrando el dispositivo de captura; C) detalle del dispositivo de captura (trampa pitfall con conservante y cebo sólido); D) EMS instalada.

Fig. 2.- Subterranean Sampling Devices (SSD). A) External aspect; B) longitudinal section of the tube showing the capture device; C) capture device detail (pitfall trap with preservative and solid bait); D) SSD installed. la península ibérica, hasta completar la instalación de 143 EMS, repartidas del siguiente modo (Fig. 3A): 16 en los Montes Cantábricos, 8 en Prepirineos, 13 en el Sistema Ibérico, 37 en el Sistema Central, y 69 en el Sistema Bético. Los enclaves seleccionados responden a un enfoque ecosistémico y geográfico, bajo el que se está obteniendo, poco a poco, información sobre artrópodos del subsuelo en las montañas de la mitad oriental de la península ibérica.

Los registros corológicos ya publicados que muestran cierta precisión toponímica, así como los de captura propia (nuevas citas) se listan en la Tabla 1, asignándoles la correspondiente coordenada UTM de $10 \times 10 \mathrm{~km}$. A partir de estas coordenadas, y con el software QGIS versión 2.16.3 (Quantum GIS Development Team, 2016), se elabora el mapa de distribución de la especie (Fig. 3A). Los nuevos muestreos proporcionaron un total de 28 especímenes (10

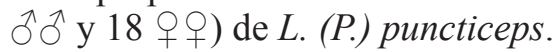

\section{Resultados y discusión}

La forma del edeago y, muy especialmente, de la lámina apical del lóbulo medio, facilita el estudio taxonómico del género Leistus. Esto no sucede con otros Nebriini, como por ejemplo las especies del género Nebria Latreille, 1802, cuyos edeagos son extraordinariamente parecidos. Por lo tanto, más allá de la consideración de otros rasgos morfológicos, el estudio de estos caracteres genitales resultó determinante para asignar las nuevas capturas ibéricas, que se presentan en este trabajo (Tabla 1) a la especie $L$. (P.) puncticeps (Figs. 1A-B, 3). Al mismo tiempo, se pone de relieve cierta variabilidad, muy leve, en la forma de la lámina apical del edeago que, en el caso de los especímenes de las localidades más meridionales, muestran el borde algo truncado, frente a las láminas apicales ligeramente más acuminadas observadas en especímenes del Moncayo (Fig. 3).

Tabla 1.- Datos con precisión toponímica de la presencia de Leistus (Pogonophorus) puncticeps en la península ibérica.

Table 1.- Data with toponymic precision of the presence of Leistus (Pogonophorus) puncticeps in the Iberian Peninsula.

\begin{tabular}{|c|c|c|c|c|}
\hline PROVINCIA & LOCALIDAD & Hábitat (colecta) & UTM & $\begin{array}{l}\text { Fuente de la } \\
\text { información }\end{array}$ \\
\hline \multirow[t]{3}{*}{ ALBACETE } & Cañada de los Mojones & $\begin{array}{l}\text { Epigeo } \\
\text { (sublapidicola })= \\
\text { E (sla) }\end{array}$ & $30 \mathrm{SWH} 45$ & $\begin{array}{l}\text { Andújar et al., } \\
2000\end{array}$ \\
\hline & Los Chorros del Río Mundo (Riópar) & E (sla) & $30 \mathrm{SWH} 45$ & $\begin{array}{l}\text { Andújar et al., } \\
2000\end{array}$ \\
\hline & $\begin{array}{l}1 \text { 万. Pico Argel, Calar del Mundo, Riópar, } 1698 \text { m., } \\
\text { 06-07-2002, V.M. Ortuño leg. }\end{array}$ & E (sla) & 30SWH55 & Nueva cita \\
\hline
\end{tabular}


Table 1. - (Continued)

\begin{tabular}{|c|c|c|c|c|}
\hline PROVINCIA & LOCALIDAD & Hábitat (colecta) & UTM & $\begin{array}{l}\text { Fuente de la } \\
\text { información }\end{array}$ \\
\hline \multirow[t]{5}{*}{ ALICANTE } & 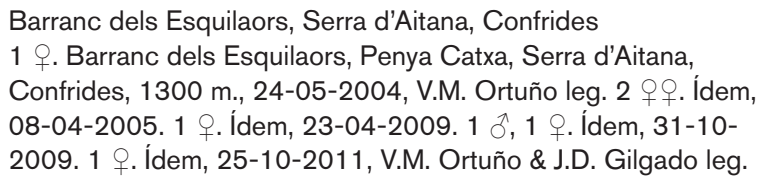 & $\begin{array}{l}\text { E (sla) e } \\
\text { hipogeo (MSS) = } \\
\mathrm{H} \text { (mss) }\end{array}$ & 30SYH38 & $\begin{array}{l}\text { Jiménez- } \\
\text { Valverde et al., } \\
2015 \\
\text { y nueva cita }\end{array}$ \\
\hline & $\begin{array}{l}1 \text { q. Mirador de Pilatos, Font Roja, Serra del Menejador, Alcoi, } \\
1180 \text { m., 23-05-2011, V.M. Ortuño leg. }\end{array}$ & $E(s l a)$ & 30SYH18 & Nueva cita \\
\hline & 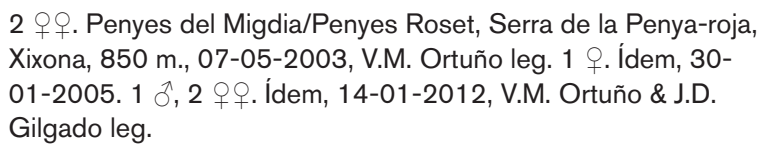 & $E$ (sla) y E (pitfall) & 30SYH17 & Nueva cita \\
\hline & $\begin{array}{l}3 \text { 웅. Serra de Bèrnia, Xaló, } 890 \text { m., 29-01-2012, V.M. Ortuño } \\
\text { \& J.D. Gilgado leg. }\end{array}$ & $H$ (mss) & 30SYH58 & Nueva cita \\
\hline & $\begin{array}{l}1 \text { Q. Teixera, Serra de Mariola, Agres, } 1160 \text { m., 16-01-2012, } \\
\text { V.M. Ortuño \& J.D. Gilgado leg. }\end{array}$ & $\mathrm{H}$ (mss) & 30SYH19 & Nueva cita \\
\hline ALMERÍA & $\begin{array}{l}1 \text { đ. Umbría de la Virgen, Sierra de Maria, María, } 1660 \text { m., } \\
\text { 17-07-2018/23-09-2018, CECOUAL leg. } 1 \text { đ. İdem, } 1666 \text { m. } \\
2 \delta^{\lambda}, 1 \text { q. Ídem, } 1660 \text { m., 29-05-2019. }\end{array}$ & $\begin{array}{l}\text { H (mss) y } \\
\text { Epigeo (coluvial) }\end{array}$ & 30SWG77 & Nueva cita \\
\hline CUENCA & Valdecabras & - & 30TWK84 & Jeanne, 1973 \\
\hline \multirow[t]{2}{*}{ HUESCA } & Puerto de Bujaruelo & - & 30TYN43 & Jeanne, 1973 \\
\hline & Puerto de Cotefablo & - & 30TYN22 & Jeanne, 1973 \\
\hline \multirow[t]{2}{*}{ LLEIDA } & Sierra de Boumort & - & 31TCG47 & Jeanne, 1973 \\
\hline & Viella (Valle de Arán) & - & $31 \mathrm{TCH} 13$ & Jeanne, 1973 \\
\hline \multirow[t]{4}{*}{ SORIA } & Barranco Peñas Negras (Cueva de Ágreda) & $\begin{array}{l}\text { Epigeo (pratícola) } \\
=E(p)\end{array}$ & 30TWM92 & Gimeno, 1984 \\
\hline & Barranco de Peñarrajada (Ágreda) & $\begin{array}{l}\text { Epigeo (hojarasca) } \\
=E(h)\end{array}$ & 30TWM93 & Gimeno, 1984 \\
\hline & Cascarrera Negra (Beratón) & $E(p)$ & зотWM92 & Gimeno, 1984 \\
\hline & Fuente de las Peñas Negras (Cuevas de Ágreda) & $E(p)$ & зотWM92 & Gimeno, 1984 \\
\hline TERUEL & Beceite & - & 31TBF62 & Jeanne, 1973 \\
\hline \multirow[t]{14}{*}{ ZARAGOZA } & Barranco Bellido (Añón) & $E(h)$ & зотХM02 & Gimeno, 1984 \\
\hline & $\begin{array}{l}\text { Barranco de Castilla (Tarazona) } \\
1 \text { q. Barranco de Castilla, Tarazona, } 1424 \text { m., 05/06/2012/25- } \\
\text { 09-2012, V.M. Ortuño, J.D. Gilgado, E. Cuesta \& E. Ledesma } \\
\text { leg. } 1 \text { J. İdem, } 1423 \text { m., 11/07/2013/23-10-2013. }\end{array}$ & $\begin{array}{l}E(h) y \\
H(\text { mss })\end{array}$ & 30TWM92 & $\begin{array}{l}\text { Gimeno, } 1984 \text { y } \\
\text { nueva cita }\end{array}$ \\
\hline & Camino del Santuario (Tarazona) & $E(h)$ & зотWM92 & Gimeno, 1984 \\
\hline & Cerro del Morrón (Añón) & $E(p)$ & зоTXM02 & Gimeno, 1984 \\
\hline & Circo de San Miguel (Tarazona) & $E(h)$ & зотWM92 & Gimeno, 1984 \\
\hline & $\begin{array}{l}\text { Colladillo Bellido (Añón) } \\
1 \text { J. Collado Bellido, Añón de Moncayo, } 1534 \text { m., } \\
\text { 05/06/2012/25-09-2012, V.M. Ortuño, J.D. Gilgado, E. Cuesta } \\
\text { \& E. Ledesma leg. } 1 \text { đ. İ́dem, 11/07/2013/23-10-2013. }\end{array}$ & $\begin{array}{l}E(h) y \\
H(\text { mss })\end{array}$ & 30TXM02 & $\begin{array}{l}\text { Gimeno, } 1984 \text { y } \\
\text { nueva cita }\end{array}$ \\
\hline & Circo del Cucharón (Tarazona) & $E(p)$ & зотWM92 & Gimeno, 1984 \\
\hline & Embalse de Morca (Añón) & $\begin{array}{l}\text { E (h) y Epigeo } \\
\text { (subcorticicola) }\end{array}$ & 30TXM02 & Gimeno, 1984 \\
\hline & Fuente de Los Frailes (Tarazona) & $E(h)$ & зотWM92 & Gimeno, 1984 \\
\hline & La Muela de Horcajuelo (Añón) & $E(p)$ & зотХM02 & Gimeno, 1984 \\
\hline & Peña Nariz (Tarazona) & $E(h)$ & 30TWM92 & Gimeno, 1984 \\
\hline & Peñas Meneras (Tarazona) & $E(h)$ & зотWM92 & Gimeno, 1984 \\
\hline & Pico Lobera (Añón) & $E(h)$ & зотWM92 & Gimeno, 1984 \\
\hline & Pista de los Bueyes (Tarazona) & $E(h)$ & зотWM92 & Gimeno, 1984 \\
\hline
\end{tabular}




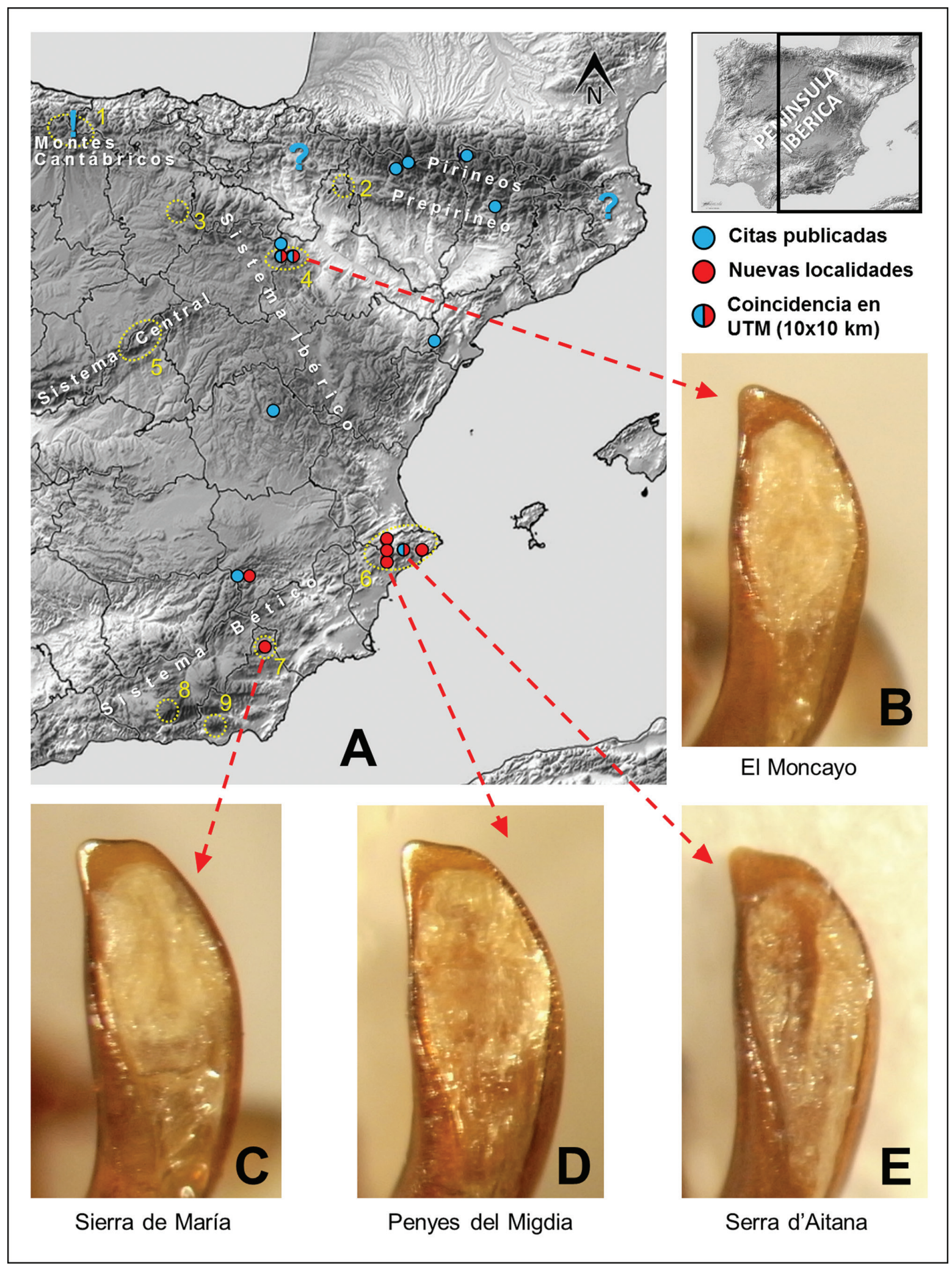

Fig. 3.-A) Áreas muestreadas con EMS (1- Montes Cantábricos centrales; 2- Sierra de Santo Domingo; 3- Sierra de Mencilla; 4- Sierra del Moncayo; 5- Sierra de Guadarrama; 6- sierras septentrionales de Alicante; 7- Sierra de María; 8- Sierra Nevada; 9- Sierra de Gádor) y localidades (publicadas y nuevas citas) con detección de Leistus (Pogonophorus) puncticeps; B) visión dorsal del lóbulo medio del edeago de Leistus (Pogonophorus) puncticeps de El Moncayo (Zaragoza); C) ídem de Sierra de María (Almería); D) ídem de Penyes del Migdia/Penyes Roset (Alicante); E) ídem de Serra d'Aitana (Alicante).

Fig. 3.- A) Sampled areas with SSD (1- Montes Cantábricos centrales; 2- Sierra de Santo Domingo; 3- Sierra de Mencilla; 4- Sierra del Moncayo; 5- Sierra de Guadarrama; 6- sierras septentrionales de Alicante; 7- Sierra de María; 8- Sierra Nevada; 9- Sierra de Gádor) and locations (published and new records) with detection of Leistus (Pogonophorus) puncticeps; B) middle lobe of the aedeagus in dorsal view of Leistus (Pogonophorus) puncticeps from El Moncayo (Zaragoza); C) ídem from Sierra de María (Almería); D) ídem from Penyes del Migdia/Penyes Roset (Alicante); E) ídem from Serra d'Aitana (Alicante). 
Los registros de Leistus (P.) puncticeps fueron interpretados, durante muchos años, como las poblaciones más occidentales (ibéricas) de Leistus (Pogonophorus) montanus Stephens, 1827. Así, con este nombre aparece registrado en las primeras publicaciones que recogen esta especie eminentemente ibérica (Fuente, 1918; Jeanne, 1966, 1973). Años más tarde, Jeanne \& Zaballos (1986) y Zaballos \& Jeanne (1994) la tratan como subespecie de L. (P.) montanus. Más recientemente, y con un mejor conocimiento taxonómico y corológico de L. (P.) montanus (sensu lato), esta especie de amplia distribución europea ha sido considerada como un pequeño complejo de especies (Farkač \& Fassati, 1999): L. (P.) montanus (sensu stricto), L. (P.) puncticeps y Leistus (Pogonophorus) parvicollis Chaudoir, 1869. Dos de ellas, $L$. (P.) montanus y $L$. $(P)$. parvicollis se extienden por gran parte de Europa (Farkač \& Janata, 2003; Vigna Taglianti, 2013), la primera es típicamente centroeuropea (Islas Británicas, Francia continental, Suiza, Italia continental, Eslovenia, Austria, Alemania, Eslovaquia, República Checa, Polonia, noroeste de Rusia), mientras que la segunda se extiende por las regiones euromediterráneas orientales (Italia continental, Eslovenia, Croacia, Bosnia Herzegovina, Bulgaria, Macedonia, Albania, Grecia continental y el archipiélago de las Cícladas y Creta) hasta llegar a Turquía y Siria. La tercera, $L$. (P.) puncticeps, puede considerarse un endemismo ibérico, si bien excede los límites de la península. Muestra una corología que perfila una distribución discontinua en la mitad oriental de España, y en algunos enclaves del sur y este de Francia (Serrano, 2003, 2013; Farkač \& Janata, 2003; Coulon et al., 2011). La distribución ibérica conocida hasta el momento de esta especie (Fig. 3A), la sitúa en el cuadrante nororiental de la península, con la excepción de contados registros en el suroeste de Albacete (Andújar et al., 2000) y en el norte de Alicante (Jiménez-Valverde et al., 2015). Más allá de las citas precisas que hasta el momento se han publicado (recogidas en la Tabla 1), también se ha referido esta especie, sin mayor exactitud, de Girona (Baehr, 1986), Altos Pirineos (sic !), Pirineos orientales (sic !), Navarra y Zaragoza (Fuente, 1918). La presencia de esta especie en Picos de Europa, citada a partir de la colecta de un espécimen (Gutiérrez et al., 2004), a nuestro juicio debe responder a un error de identificación, tratándose muy probablemente de Leistus (Pogonophorus) spinibarbis (Fabricius, 1775), si bien no podemos afirmarlo categóricamente.

Se dispone de poca información sobre el tipo de hábitat en donde vive $L$. (P.) puncticeps, no obstante, se puede hacer algún tipo de inferencia a partir del conocimiento de su especie hermana, L. (P.) montanus, generalmente presente en pedregales de ladera y depósitos de rocas (Luff, 1998), y por ello es calificada como petrófila, aunque también ha sido observada en praderas alpinas (Fritze \& Hannig, 2010). Cabría esperar lo mismo en $L$. (P.) puncticeps, y lo publicado hasta el momento así parece confirmarlo, al menos en parte, pues Jeanne (1973) indica que muestra predilección por hábitats pedregosos, en parajes quebrados y abruptos. En el Moncayo parece estar presente en este mismo tipo de suelos, tanto en la franja forestal (hayedos, robledales y pinares) como en la supraforestal (enebro rastrero y pastizales montanos) (Gimeno, 1984). Las nuevas localidades que aquí se aportan confirman la presencia, nada anecdótica, de $L$. (P.) puncticeps en algunos relieves del Sistema Bético: en la cordillera Prebética (Alicante y Albacete), y el registro más meridional en la cordillera Subbética (Almería) (Fig. 3A). Desde una perspectiva puramente biogeográfica, la distribución de L. (P.) puncticeps se ve ampliada de forma muy clara a enclaves de la región Mediterránea, concretamente al sur de la provincia Catalano-Valenciano-Provenzal, y en la zona de confluencia de las provincias Bética y CastellanoMaestrazgo-Manchega (Fig. 4A). Estas nuevas localidades cuentan, en general, con precipitación escasa y torrencial, si bien se hallan próximas a áreas con una pluviosidad media que ronda los 600-700 mm (Fig. 4B), con la excepción de Sierra de María, y algún enclave de las sierras de Alicante (Serra de la Penyaroja con 240 a $380 \mathrm{~mm}$ de precipitación media anual, -ver Padilla Blanco, 1995-) que son manifiestamente más xéricos. Por el contrario, otros enclaves con una peculiar orografía y próximos a la costa, como la Serra de Bèrnia, promueven el desarrollo de lluvias locales (Morales et al., 1983) y criptoprecipitación, mostrándose como refugios adecuados para cierta fauna estenohigrobia. Respecto a las temperaturas, estas áreas montañosas experimentan una notable amplitud térmica. Un buen ejemplo de ello es la Serra d'Aitana que, en verano, llega a superar $\operatorname{los} 30^{\circ} \mathrm{C}$, mientras que en invierno nieva con regularidad, como pone en evidencia las antiguas construcciones de pozos de nieve que se hallan en la zona (Vicedo \& Ramírez, 2004). A tenor de lo expuesto, se explica que determinadas especies estenotermas, de ambientes fríos, también hayan encontrado condiciones de vida adecuadas en algunas de estas montañas mediterráneas que actúan como refugio. Este es el caso de L. (P.) puncticeps que, sobre un escenario geológico quebrado y abrupto, y bajo condiciones mínimamente adecuadas (rango altitudinal, orientación, régimen de vientos, y cantidad/naturaleza de las precipitaciones) ha sobrevivido, constituyendo poblaciones relictuales de lo que antaño fue, muy posiblemente, una distribución más homogénea. Este fenómeno también se observa con ciertas especies vegetales que sobreviven en estos relieves, como los escasos tejos, Taxus baccata L. (Solanas i Ferràndiz \& Mateo Sanz, 1991; Andrés Ros et al., 2007) y acebos, Ilex aquifolium L., que quedan en la provincia de Alicante (Crespo et al., 2007).

La presencia de L. (P.) puncticeps en el Medio Subterráneo Superficial (MSS) no puede calificarse de accidental, pues ha sido registrada en tres áreas 

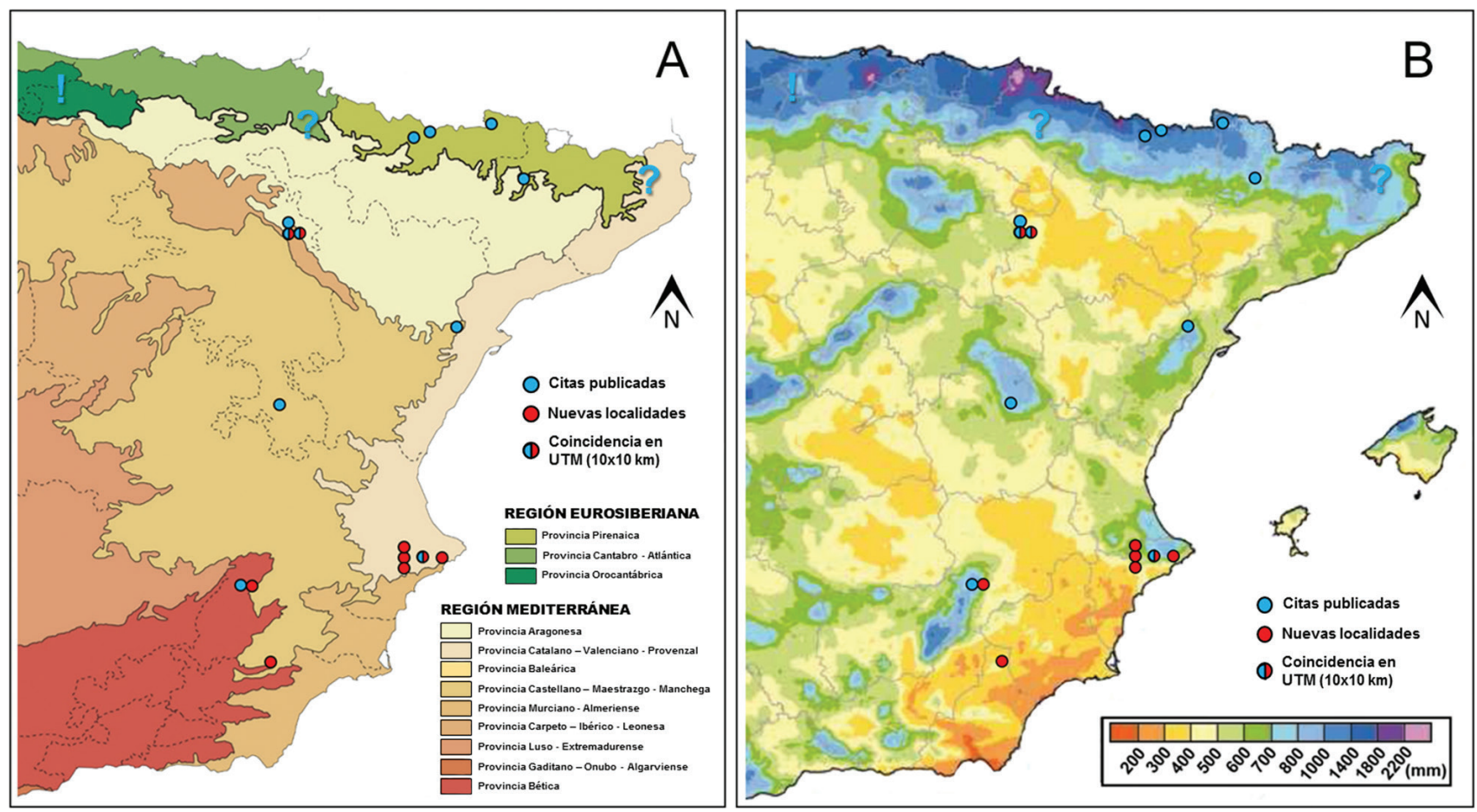

Fig. 4.- A) Distribución de Leistus (Pogonophorus) puncticeps superpuesta al mapa biogeográfico de regiones y provincias (modificado de Rivas Martínez, 1987); B) distribución de Leistus (Pogonophorus) puncticeps superpuesta al mapa pluviométrico promedio anual (modificado de AEMET \& IMP, 2011).

Fig. 4.- A) Distribution of Leistus (Pogonophorus) puncticeps superimposed to the biogeographic regions and provinces map (modified from Rivas Martínez, 1987); B) distribution of Leistus (Pogonophorus) puncticeps superimposed to the annual average pluviometric map (modified from AEMET \& IMP, 2011).

geográficas distantes unas de otras, con clima, geomorfología y litología diferentes (Sierra del Moncayo; formaciones montañosas del norte de la provincia de Alicante y Sierra de María) (Tabla 1; Figs. 3A, 5). Es evidente que en $L$. (P.) puncticeps, al menos los imagos se aventuran por el MSS, desarrollando un estilo de vida que se podría definir como subtroglófilo (sensu Sket, 2008). Este peculiar comportamiento ya ha sido observado en otras especies ápteras del género (Ortuño et al. 2019; Ortuño obser, pers.), si bien en $L$. (P.) puncticeps es singular al tratarse de una especie alada (Fig. 1B). La facultad del vuelo le confiere, a priori, un mayor potencial de dispersión (Gimeno, 1984) que a las especies ápteras (Fig. 1C), por ejemplo, del "grupo Leistus angusticollis" sensu Perrault (1979), y con las que comparte tendencia geófila e higrófila. Posiblemente sean estas dos últimas características las que promueven que $L$. (P.) puncticeps opte por buscar refugio en el subsuelo, especialmente para sobrevivir a la estación más seca. Una vez más, el MSS se revela como un hábitat en donde se desenvuelven bien especies estenoicas que se suelen mostrar activas en medios epigeos cuando los factores ambientales son favorables para la especie.

Todo lo expuesto sugiere que $L$. (P.) puncticeps ha seguido la misma historia evolutiva que $L$. (P.) montanus (sensu stricto) y L. (P.) parvicollis, a tenor de la distribución que muestran estas especies y la estrecha relación que tienen entre sí (Assmann, 1993; Farkač $\&$ Fassati, 1999), constituyendo una triada de relictos glaciales. Posiblemente, la especie ancestral de Leistus que dio origen a estas tres especies, fue desplazada durante el Último Máximo Glacial (hace 20.000 años) a los "refugios pleistocénicos" de Europa mediterránea, principalmente a tres penínsulas: ibérica, itálica y balcánica. Las poblaciones ancestrales del actual "complejo de L. (P.) montanus", sufrieron especiación por vicarianza, en cada uno de los tres grandes refugios, originándose así L. (P.) puncticeps, L. (P.) montanus y $L$. (P.) parvicollis. Ya en el período postglacial, los dos últimos se expandieron de forma notable, más allá de las penínsulas, mientras que $L$. (P.) puncticeps experimentó una expansión mucho más limitada, rebasando los Pirineos hasta alcanzar algunos relieves de la mitad sur y este de Francia. La falta de registros de esta especie en la mitad occidental de la península ibérica, sugiere que sus ancestros nunca llegaron a establecerse en esta amplia zona. Se observa que la distribución de $L$. (P.) puncticeps coincide, en gran medida, con el área de menor variación climática extrema postglacial, según el modelo del índice de precipitación estándar (SPI) desarrollado por Burke et al. (2014). Estas áreas, halladas en su mayoría en la mitad oriental de la península, fueron las más idóneas para el asentamiento de las especies según estos autores, pues fueron mucho menos áridas que la mitad occidental ibérica, al no 

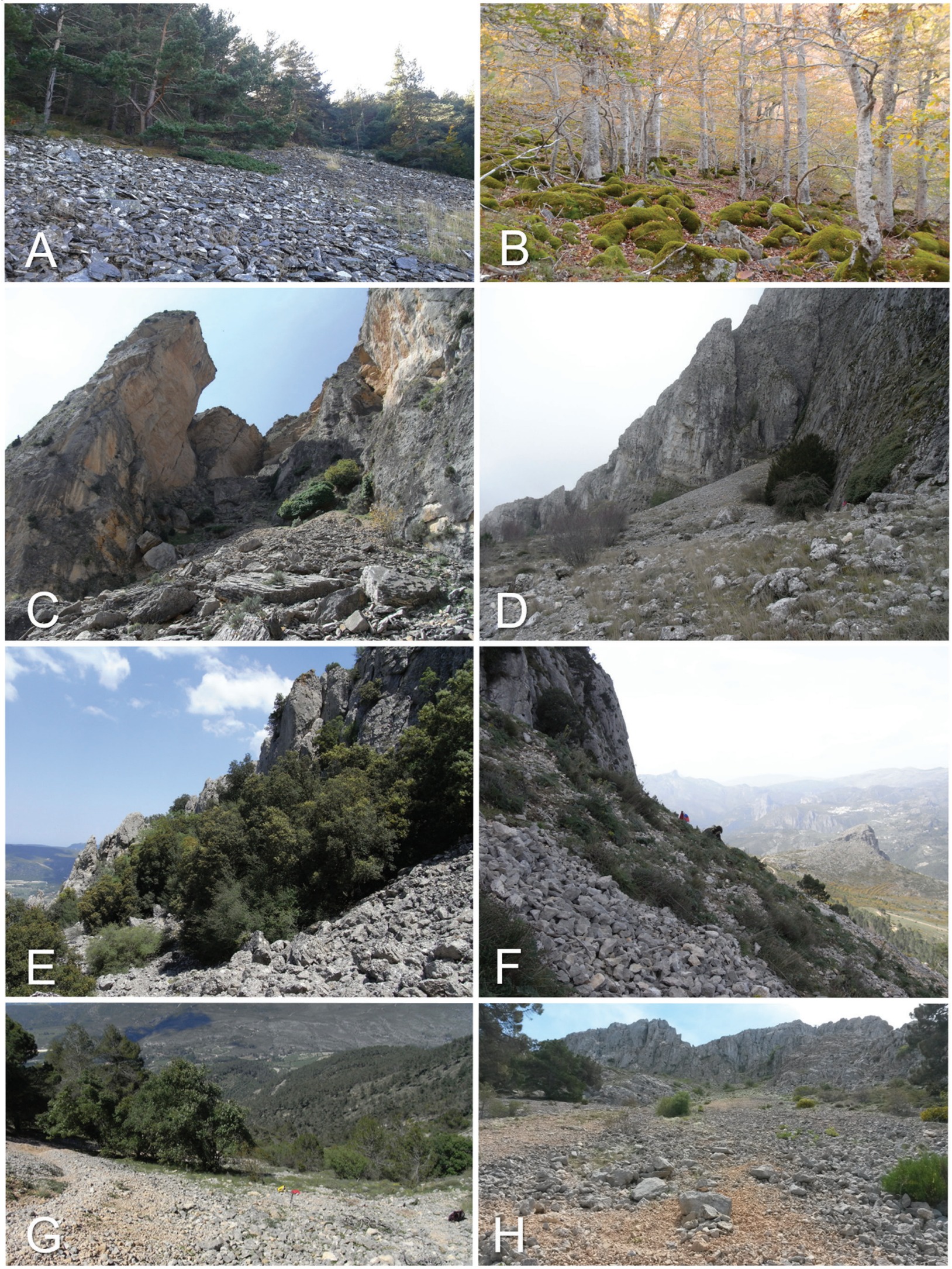

Fig. 5.- Hábitats de Leistus (Pogonophorus) puncticeps: A) Sierra del Moncayo (Collado Bellido); B) Sierra del Moncayo (Barranco de Castilla); C) Penyes del Migdia/Penyes Roset, Serra de la Penya-roja; D) Barranc dels Esquilaors, Penya Catxa, Serra d'Aitana; E) Font Roja, Serra del Menejador; F) Serra de Bèrnia; G) Teixera, Serra de Mariola; H) Umbría de la Virgen, Sierra de María.

Fig. 5.- Habitats of Leistus (Pogonophorus) puncticeps: A) Sierra del Moncayo (Collado Bellido); B) Sierra del Moncayo (Barranco de Castilla); C) Penyes del Migdia/Penyes Roset, Serra de la Penya-roja; D) Barranc dels Esquilaors, Penya Catxa, Serra d'Aitana; E) Font Roja, Serra del Menejador; F) Serra de Bèrnia; G) Teixera, Serra de Mariola; H) Umbría de la Virgen, Sierra de María. 
estar afectadas por sequías y heladas extremas. Con el trascurso de los milenios, la elevación de las temperaturas supuso la paulatina reducción en el rango de distribución de especies calificadas como relictos glaciales, entre ellas $L$. (P.) puncticeps. El conjunto de pulsos climáticos sobrevenidos desde el Último Máximo Glacial, explicaría la distribución discontinua que muestra en la actualidad $L$. (P.) puncticeps. $\mathrm{Su}$ presencia en el MSS de los enclaves relictuales, responde a la naturaleza estenoica de estos carábidos, a su higrofilia y a su especialización en la depredación de Collembola y Acari, artrópodos muy abundantes en el subsuelo (Ortuño et al., 2019).

Un mejor y más amplio estudio de la fauna de las montañas ibéricas, así como de los hábitats subterráneos que en ellas se han desarrollado, contribuiría a: 1) perfilar con más precisión las áreas de distribución de especies orófilas con hábitos geófilos; 2) conocer mejor los aspectos autoecológicos de estas especies y, con ello, caracterizar los hábitats que ocupan.

\section{Agradecimientos}

Son muchas las personas que durante estas últimas décadas han colaborado, de un modo u otro, en los innumerables muestreos que se han realizado. Sería muy largo el listado de nombres que debería citarse aquí por lo que, en aras de la brevedad, tomamos la decisión de dejarlos en el anonimato. Si alguno de ellos lee este artículo, podrá reconocer cuánto contiene de su esfuerzo de muestreo y, con él, también esperamos que perciba nuestro silente pero emocionado agradecimiento. Son muchas las instituciones que, de un modo u otro, también nos han brindado su apoyo. Uno de los autores (A.J.-V.) disfruta de un contrato del programa Ramón y Cajal (RYC-2013-14441) del Ministerio de Ciencia, Innovación y Universidades.

\section{Referencias}

AEMET \& IMP, 2011. Atlas Climático Ibérico. Agencia Estatal de Meteorología, Ministerio de Medio Ambiente y Medio Rural y Marino e Instituto de meteorología de Portugal.

Andrés Ros, J. V., Fabregat, C., López Udias, S., Aparicio, J. M., Prada, A., Martínez Llistó, J., García Martí, X., Serena, V., López Martos, J., Herreros, R., Marzo, A., Cerdán, V., Bayarri, X., Bosch, F., Gómez Talens, J. \& Zreik, C., 2007. Censos y caracterizaciones de tejedas de la Comunidad Valenciana. I Jornadas sobre el tejo en el Mediterráneo Occidental: 127-136.

Andújar, A., Lencina, J.L., Ruano, L. \& Serrano, J., 2000. Los Caraboidea de las sierras suroccidentales de la provincia de Albacete. Instituto de Estudios Albacetenses "Don Juan Manuel”. Albacete.

Assmann, Th., 1993. Die Leistus-Arten der Oreobius-Gruppe: Systematik, Taxonomie und Verbreitung (Coleoptera, Carabidae: Nebriinae). Mitteilungen aus der Entomogischen Gesellschaft Basel, 43(2): 42-68.

Baehr, M., 1986. On the carabid fauna of the province of Girona, Northeastern Spain (Insecta, Coleoptera). Miscel-lània Zoològica, 10: 161-171.
Burke, A., Levavasseur, G., James, P. A. M., Guiducci, D., Izquierdo, M. A., Bourgeon, L., Kageyama, M., Ramstein, G. \& Vrac, M., 2014. Exploring the impact of climate variability during the Last Glacial Maximum on the pattern of human occupation of Iberia. Journal of Human Evolution, 73: 35-46. https://doi.org/10.1016/j. jhevol.2014.06.003

Coulon, J., Pupier, R., Queinnec, E., Ollivier, E. \& Richoux, P., 2011. Coléoptères Carabidae de France. Complément et mise à jour. Volume 1. Faune de France 94. Fédération française des Sociétés de Sciences naturelles. Paris.

Crespo, M. B., Camuñas. E. \& Cristóbal, J. C., 2007. Precisiones corológicas y taxonómicas sobre la flora de Alicante. Flora Montiberica, 36: 52-64.

Farkač, J. \& Fassati, M., 1999. Subspecific taxonomy of Leistus montanus from Central Europe (Coleoptera: Carabidae: Nebriini). Acta Societatis Zoologicae Bohemoslovacae, 63: 407-425.

Farkač, J. \& Janata, M., 2003. Nebriini, In: I. Löbl \& A. Smetana (eds.). The Catalogue of Palaearctic Coleoptera. Vol. 1. Apollo Books, Stenstrup, Denmark: 79-96.

Fritze, M.-A. \& Hannig, K., 2010. Verbreitung und Ökologie von Leistus montanus Stephens, 1827 in Deutschland (Coleoptera: Carabidae). Angewandte Carabidologie, 9: 39-50.

Fuente, de la, J.M., 1918. Catalogo sistemático-geográfico de los coléopteros observados en la Península Ibérica, Pirineos propiamente dichos y Baleares. Boletín de la Sociedad Entomológica de España, 1(9): 178-193.

Gers, C., 1998. Diversity of energy fluxes and interactions between arthropod communities: from soil to cave. Acta Oecologica, 19: 205-213. https://doi.org/10.1016/ S1146-609X(98)80025-8

Giachino, P. M. \& Vailati, D., 2017. Considerations on biological and terminological aspects of the subterranean and endogean environments: diversity, correlations and faunistic interchange. Atti Accademia Nazionale Italiana di Entomologia, 65: 157-166.

Gimeno, J.A., 1984. Los Carabidae (Coleoptera Adephaga) de la Sierra del Moncayo. Tesis Doctoral. Universidad Complutense. Madrid.

Gutiérrez, D., Menéndez, R. \& Méndez, M., 2004. Habitat-based conservation priorities for carabid beetles within the Picos de Europa National Park, northern Spain. Biological Conservation, 115(3): 379-393. https://doi.org/10.1016/S0006-3207(03)00154-X

Jeanne, C., 1966. Carabiques de la Péninsule ibérique (3e note). Actes de la Société linnéenne de Bordeaux, série A, 103(7): 3-18.

Jeanne, C., 1973. Carabiques de la Péninsule ibérique (1er supplement). Bulletin de la Société Linnéenne de Bordeaux, 3(1): 3-20.

Jeanne, C. \& Zaballos, J.P., 1986. Catalogue des Coléoptères Carabiques de la Péninsule Ibérique. Société Linnéenne de Bordeaux, Supplément au Bulletin de la Société Linnéenne de Bordeaux, Bordeaux, France.

Jiménez-Valverde, A., Gilgado, J. D., Sendra, A., PérezSuárez, G., Herrero-Borgoñón, J. J. \& Ortuño, V. M., 2015. Exceptional invertebrate diversity in a scree slope 
in Eastern Spain. Journal of Insect Conservation, 19(4): 713-728. https://doi.org/10.1007/s10841-015-9794-1

Juberthie, C., 2000. The diversity of the karstic and pseudokarstic hypogean habitats in the world, In: H. Wilkens, D.C. Culver \& W.F. Humphreys (eds.). Subterranean Ecosystems. Elsevier Press. Amsterdan: 17-39.

Juberthie, C., Bouillon, M. \& Delay, B., 1981. Sur l'existence du milieu souterrain superficiel en zone calcaire. Mémoires de Biospéologie, 8: 77-93.

Juberthie, C., Delay, D. \& Bouillon, M., 1980. Extension du mille souterrain en zone non calcaire: description d'un nouveau milieu et de son peuplement par les Coléoptères troglobies. Mémoires de Biospéologie, 7: 19-52.

Luff, M.L., 1998. Provisional atlas of the ground beetles (Coleoptera, Carabidae) of Britain. Biological Records Centre. Huntingdon.

Mammola S., Giachino, P. M., Piano, E., Jones, A., Barberis, M., Badino, G. \& Isaia, M., 2016. Ecology and sampling techniques of an understudied subterranean habitat: the Milieu Souterrain Superficiel (MSS). The Science of Nature, 103: 88. https://doi.org/10.1007/s00114-016-1413-9

Morales Gil, A., Bru Ronda, C. \& Box Amorós, M., 1983. Morfología en la Umbría de la Sierra de Bernia. Investigaciones Geográficas, 1: 117-146.

Moseley, M., 2010. Are all caves ecotones? Cave and Karst Science, 36(2009): 53-58.

Oromí, P., Medina, A. L. \& Tejedor, M. L., 1986. On the existence of a superficial underground compartment in the Canary Islands. Acta IX Congreso Internacional de Espeleología, 2: 147-151.

Ortuño, V. M., Gilgado, J. D., Jiménez-Valverde, A., Sendra, A., Pérez-Suárez, G. \& Herrero-Borgoñón, J.J., 2013. The "Alluvial Mesovoid Shallow Substratum", a new subterranean habitat. PLoS ONE, 8(10), e76311. https://doi.org/10.1371/journal.pone.0076311

Ortuño, V. M., Ledesma, E., Jiménez-Valverde, A. \& PérezSuárez, G., 2019. Studies of the mesovoid shallow substratum can change the accepted autecology of species: the case of ground beetles (Coleoptera: Carabidae) in the Sierra de Guadarrama National Park (Spain). Animal Biodiversity and Conservation, 42(2): 213-226. https://doi.org/10.32800/abc.2019.42.0213

Padilla Blanco, A., 1995. El poblamiento vegetal en las sierras de Peñarroya y Cuartel: aspectos evolutivos y situación actual. Universidad de Alicante, Alicante, e Instituto Universitario de Geografía, Compobell S.L., Murcia.
Perrault, G.-G., 1979. Le genre Leistus (Froehlig) (Coleoptera - Carabidae). I. Le groupe de Leistus angusticollis (Dejean). Bulletin Mensuel de la Societe Linnéenne de Lyon, 48(1): 53-64.

Pipan, T., López, H., Oromí, P., Polak, S. \& Culver, D. C., 2011. Temperature variation and the presence of troglobionts in terrestrial shallow subterranean habitats. Journal of Natural History, 45(3-4): 253-273. https://doi.org/10. $1080 / 00222933.2010 .523797$

Quantum GIS Development Team, 2016. Quantum GIS Geographic Information System. Open Source Geospatial Foundation Project. http://qgis.osgeo.org

Rivas Martínez, S., 1987. Memoria del mapa de series de vegetación de España. 1:400 000. ICONA, Serie técnica.

Serrano, J., 2003. Catálogo de los Carabidae (Coleoptera) de la Península Ibérica. Monografías S.E.A. 9. Sociedad Entomológica Aragonesa. Zaragoza.

Serrano, J., 2013. Nuevo catálogo de la familia Carabidae de la península ibérica ("Coleoptera"). Universidad de Murcia. Murcia.

Sket, B., 2008. Can we agree on an ecological classification of subterranean animals? Journal of Natural History, 42(21-22): 1549-1563. https://doi.org/10. $1080 / 00222930801995762$

Solanas i Ferràndiz, J. L. \& Mateo Sanz, G., 1991. Plantes de la serra de la Serrella (El Comtat-La Marina Baixa). Butlletí de la Institució Catalana d'Història Natural, 59(8): 75-79.

Uéno, S. I., 1980. The anophthalmic trechine beetles of the group of Trechiama ohshimai. Bulletin of the National Science Museum, Tokyo, Serie A, 6(4): 195-274.

Uéno, S. I., 1981. New anophthalmic Trechiama (Coleoptera, Trechinae) from northern Shikoku, Japan. Journal of the speleological Society of Japan, 6: 11-18.

Vicedo, M. \& Ramírez, J., 2004. Guía de los Pozos de Nieve de la Provincia de Alicante. Diputación de Alicante. Alicante.

Vigna Taglianti, A., 2013. Fauna Europaea: Carabidae. In: P. Audisio, 2019. Fauna Europaea: Coleoptera. Fauna Europaea version 2017.06, https://fauna-eu.org

Zaballos, J. P. \& Jeanne, C., 1994. Nuevo catálogo de los carábidos (Coleoptera) de la Península Ibérica. Monografías de la Sociedad Entomológica Aragonesa, 1: 1-159. 